\title{
AMBITO DE HOGAR DE LIOLAEMUS MELANOPS BURMEISTER, 1888 (SQUAMATA: LIOLAEMINI) EN EL CENTRO DE CHUBUT, ARGENTINA
}

\author{
HOME RANGE OF LIOLAEMUS MELANOPS BURMEISTER, 1888 \\ (SQUAMATA: LIOLAEMINI) IN CENTRAL CHUBUT, ARGENTINA
}

\author{
Nicolás Frutos ${ }^{1}$, Leonardo A. Camporro ${ }^{2} \&$ Luciano J. Avila $^{1}$ \\ ${ }^{1}$ Centro Nacional Patagónico (CENPAT-CONICET). Bv. Brown 2825. U9120ACF \\ Puerto Madryn - Chubut. Argentina. frutos@cenpat.edu.ar \\ ${ }^{2}$ Universidad Nacional de la Patagonia San Juan Bosco. Bv. Brown 3700. U9120ACV \\ Puerto Madryn - Chubut. Argentina.
}

\begin{abstract}
RESUMEN
Liolaemus melanops es un lagarto de mediano tamaño (hasta $90 \mathrm{~mm}$ LHC), que habita zonas arenosas bajas y dunas costeras en las provincias argentinas de Río Negro y Chubut. En este estudio se analizó el ámbito de hogar de una población de L. melanops en el centro de Chubut, durante el período febrero a mayo de 2005. En una cuadrícula de 100 x $100 \mathrm{~m}$, todos los individuos observados fueron capturados, sexados, medidos, marcados y liberados en el mismo sitio. Mediante transectas de rumbo fijo, realizadas a intervalos regulares entre las 8:00 y las 19:00 h, fue registrada la posición de todos los individuos observados. El ámbito de hogar de cada ejemplar fue calculado utilizando el método del polígono convexo mínimo. El área promedio para los machos no fue significativamente diferente al de las hembras. No se encontró correlación entre el tamaño corporal y el tamaño del ámbito de hogar. Se observó un gran solapamiento en el ámbito de hogar en machos pero no en hembras. Estos resultados se discuten a la luz de la teoría actual.
\end{abstract}

Palabras claves: Liolaemus, Patagonia, Polígono Convexo Mínimo, Liolaemini.

\section{ABSTRACT}

Liolaemus melanops is a medium sized lizard (up to $90 \mathrm{~mm} \mathrm{SVL}$ ) that inhabits sandy lowlands and coastal dunes in Río Negro and Chubut Provinces. We studied the home range of a population of Liolaemus melanops from central Chubut Province between February and May, 2005. In a grid of $100 \times 100 \mathrm{~m}$ all observed individuals were captured, sexed, measured, marked and released. We made regular transects between 08:00 and 19:00 $\mathrm{h}$ and registered the position of all observed lizards. Home range was calculated using the Minimum Convex Polygon method. No differences in Home range between males and females were found. There was no correlation between body size and home range. Home range overlap was low in females but high in males. These results are discussed at the light of current theory.

Keywords: Liolaemus, Patagonia, Minimum Convex Polygon, Liolaemini.

\section{INTRODUCCION}

El tamaño del ámbito de hogar es considerado como un buen indicador de los requerimientos de recursos en relación a su disponibilidad en el ambiente y de las características del comportamiento de un animal, de modo que existe un interés constante en ecología por comprender los factores que predicen el tamaño del dominio vital (Perry \& Garland 2002). Estas distribuciones espaciales son frecuentemente descriptas en términos de dominio vital, ámbito de hogar o área de acción (home range), y es definida 
como el área donde un individuo realiza sus actividades diarias (Burt 1943). Aún cuando se ha definido un territorio como la porción del ámbito de hogar que es defendida para un uso exclusivo (Brown \& Orians 1970), en su ámbito de hogar un animal puede encontrar todo lo necesario para su supervivencia (alimento, agua, lugares de anidamiento, pareja, incluyendo también refugio y condiciones térmicas adecuadas) (Stamps 1983, Christian \& Waldschmidt 1984, Huey et al. 1989, Wone \& Beauchamp 2003). La determinación en la naturaleza del ámbito de hogar se torna compleja cuando se consideran factores como la escala temporal, el sexo, la edad y otras características biológicas de los individuos (Christian \& Waldschmidt 1984, Huey et al. 1983, Rose 1982, Turner et al. 1969).

Gran parte de los estudios sobre comportamiento territorial y tamaño del ámbito de hogar realizados en saurios se ha focalizado en familias de ambientes desérticos o tropicales del hemisferio norte y Australia, principalmente en especies de los géneros Anolis, Sceloporus, Psammodromus, Podarcis, Agama y Chalcides (ver Christian \& Waldschmidt 1984, Martins 1994, Perry \& Garland 2002). En el sur de América del Sur varios estudios sobre la distribución espacial y uso del espacio se han centrado en especies de Liolaemus Wiegmann, 1834. Mientras que la información para especies chilenas es relativamente abundante (Fuentes 1981, Fuentes \& Cancino 1979, Fox \& Shipmann 2003, Habit \& Ortiz 1994, Jaksic 2001, Jaksic et al 1979, 1980, Jerez \& Ortiz 1975, Manssur \& Fuentes 1979, Ortiz 1981, Medel et al 1988, Schulte II et al 2004, Simonetti 1974, Simonetti \& Ortiz 1980, Araya Díaz 2007), los estudios en especies argentinas son escasos (e.g. Halloy 1996, Frutos 2001, Halloy \& Robles 2002). En general, los estudios enumerados anteriormente muestran que los machos presentan ámbitos de hogar mayores a los de las hembras y generalmente los de éstas últimas muchas veces se encuentran incluidos dentro de los ámbitos de hogar de los machos (e.g. Simonetti \& Ortiz 1980, Halloy 1996, Rocha 1999, Frutos 2001, Halloy \& Robles 2002).

El objetivo de este trabajo fue caracterizar el ámbito de hogar de una población de Liolaemus melanops en el centro de Chubut, Argentina y realizar un análisis preliminar de sus factores determinantes.

\section{MATERIALES Y METODOS}

Liolaemus melanops Burmeister 1888 es una especie de mediano tamaño (hasta $90 \mathrm{~mm}$ de largo hocico-cloaca), diurna, insectívora y ovípara que vive en ambientes arenosos del Monte austral (Cei 1986). El sitio de estudio está ubicado en Pampa La Jarilla, $40 \mathrm{~km}$ al sur de la localidad de Telsen (42 44' S, 66 59' W) Departamento Telsen, Provincia del Chubut, Argentina. La región esta incluida dentro de la biozona denominada como Monte de Llanuras y Mesetas (APN/SRNyDS, 1999). En esta área las precipitaciones son en general de $100 \mathrm{~mm}$ anuales llegando ocasionalmente a $200 \mathrm{~mm}$ y una temperatura media anual de $10^{\circ} \mathrm{C} \mathrm{a} 13^{\circ} \mathrm{C}$. Los suelos, en general, son arenosos profundos (Aridisoles). La vegetación está definida por matorrales donde predominan las jarillas (Larrea divaricata y L. cuneifolia), que determinan la fisonomía de la comunidad. Otras lagartijas que coexisten con L. melanops son L. darwinii (Bell 1843), Leiosaurus belli (Duméril \& Bibron 1837) y Cnemidophorus longicauda (Bell 1843).

Dentro del área de estudio se seleccionó una parcela de 100 x $100 \mathrm{~m}$. en la cual se construyó una cuadrícula ortogonal utilizando un nivel óptico automático Kern, modelo GK1A. La cuadricula fue subdividida en cuadrantes de 5 metros de lado. Con el objetivo de identificar la posición de los individuos dentro de la cuadricula cada vértice del cuadrante fue marcado por un número y una letra (Gil et al. 1989). Dentro de la cuadrícula y antes de iniciar el estudio, los ejemplares fueron capturados y marcados mediante mostacillas de colores según la metodología propuesta por Fisher \& Muth (1989). Estas mostacillas fueron suturadas a la base de la cola con una hebra de monofilamento de acero quirúrgico con una aguja hipodérmica. También fueron marcados por amputaciones de los dedos por el método de Ferner (1979). Cada sitio fue recorrido a intervalos regulares, siguiendo coordenadas establecidas. A fin de evitar errores asociados a la posición de los individuos el comienzo de cada transecta fue elegido aleatoriamente. Dos observadores, separados tres metros uno del otro realizaron los registros, lográndose así que el segundo observador pueda divisar las lagartijas que corren al paso del primero (Maury 1981). Los muestreos 
fueron realizados en días que mostraron iguales condiciones ambientales $\left(31,8 \pm 9,6^{\circ} \mathrm{C}\right)$ con esfuerzo de registro constante (Tellería 1986). Las variables registradas durante el trabajo de campo fueron: número del individuo, sexo, longitud hocico cloaca (LHC), longitud total (LT), ubicación espacial, día y hora. Al principio de cada transecta se registró la temperatura del sustrato al sol y a la sombra. Para estimar el ámbito de hogar, se registró la ubicación espacial de los individuos en la cuadrícula en relación al punto de referencia más cercano el que se calculó por medio del método de los Polígonos Convexos Mínimos (MPC) para así posibilitar comparaciones con antecedentes de la literatura (Hayne 1949). Los datos fueron analizados en Infostat/P (Infostat 2005) y Biotas $^{\mathrm{TM}}$. El MPC tiene ventajas sobre los métodos estadísticos ya que es simple de calcular incluso en el campo, es exacto con un adecuado número de observaciones y se basa en las observaciones reales del animal. Además el MPC no realiza ningún juicioa priori de cómo las lagartijas ocupan el espacio (Haenel et al. 2003a, b). Se utilizó un test t de comparaciones de medias para evaluar las diferencias en la LT, LHC y en el tamaño de los ámbitos de hogar. Se utilizó un análisis de correlación para evaluar la existencia de relaciones entre el tamaño corporal (LT y LHC) y el tamaño de los ámbitos de hogar. Para cumplir con los supuestos del modelo, los datos fueron transformados aplicando logaritmo natural (ln). Se calculo el índice de dimorfismo sexual como:

$$
\mathrm{D} \mathrm{s}=\frac{\bar{X} \text { tamaño corporal sexo más grande }}{X \text { tamaño corporal sexo más chico }}-1
$$

(Lovich \& Gibbons 1992, Watkins 1996).

\section{RESULTADOS}

Se encontraron diferencias significativas para LT entre machos (media $=16,51 \pm 1,95 \mathrm{~cm}, \mathrm{n}=28) \mathrm{y}$ hembras (media=15,07 $\pm 2,11 \mathrm{~cm}, \mathrm{n}=29)$, $(\mathrm{t}=2,66$, $\mathrm{p}=0,010$ ), así como en LHC de machos (media=7,64 \pm $0,64 \mathrm{~cm}, \mathrm{n}=28$ ) y hembras (media $=7,18 \pm 0,52 \mathrm{~cm}, \mathrm{n}=29$ ) $(\mathrm{t}=2,96, \mathrm{p}=0,0045)$. Sin embargo, el grado de dimorfismo sexual es bajo y no significativo: 0,10 (Ds de LT) y 0,06 (Ds de LHC).
De un total de 57 individuos marcados, se obtuvo el ámbito de hogar de 14 individuos (con cuatro o más observaciones por individuo). No se encontraron diferencias significativas entre los ámbitos de hogar de machos (media $=70,91 \pm 37,37 \mathrm{~m}^{2}, \mathrm{n}=6$ ) y hembras (media=42,10 $\left.\pm 51,29 \mathrm{~m}^{2}, \mathrm{n}=8\right),(\mathrm{t}=1,81, \mathrm{p}=0,095)$.

El grado de solapamiento de los ámbitos de hogar entre las hembras fue mínimo, registrándose en una sola oportunidad (Tabla I, Fig. 1). Entre machos el grado de solapamiento fue importante en algunos casos (individuos M2 y M4 en Fig. 1) en que los individuos compartieron gran parte $\left(42,04 \mathrm{~m}^{2}\right)$ de sus ámbitos de hogar. El solapamiento de ámbitos de hogar entre ambos sexos fue moderado en relación a los valores anteriores (Tabla I, Fig.1), en un caso el ámbito de hogar de un macho se solapa con el de dos hembras (macho $\mathrm{n}^{\circ} 2$ ).

No se encontró correlación entre el tamaño corporal (LT) $(r=0,41, p=0,141)$ y $(\mathrm{LHC})(\mathrm{r}=0,17, \mathrm{p}=0,550)$ y el tamaño del ámbito de hogar (Figs. 2 y 3 ).

\section{DISCUSION}

Los ámbitos de hogar de machos y hembras de Liolaemus melanops no muestran diferencias significativas en cuanto a tamaño. Este resultado se aparta de lo encontrado en L. koslowskyi en el monte de la provincia de La Rioja, Argentina (Frutos 2001), donde los machos tienen ámbitos de hogar 5 6 veces mayores que los de las hembras. Este último patrón también ha sido encontrado en otras especies argentinas y de Brasil del género (Tabla II; Halloy \& Robles 2002; Rocha 1999).

El mayor ámbito de hogar de los machos muchas veces se relacionó con el dimorfismo sexual en tamaño; bajo esta hipótesis, un mayor tamaño corporal conlleva un mayor requerimiento energético y por la necesidad de una mayor área de forrajeo (Rocha 1999). Sin embargo, machos y hembras de algunas especies que no presentan dimorfismo sexual sí presentan diferencias en el tamaño de sus ámbitos de hogar (Stamps 1983). Algunos autores (e.g., Perry \& Garland 2001) han sugerido que el tamaño del ámbito de hogar en las hembras es determinado primariamente por necesidades energéticas, mientras que en machos el mismo sería influenciado en mayor medida por factores sociales (mayor ámbito de hogar implicaría mayor disponibilidad de hembras para aparearse). 


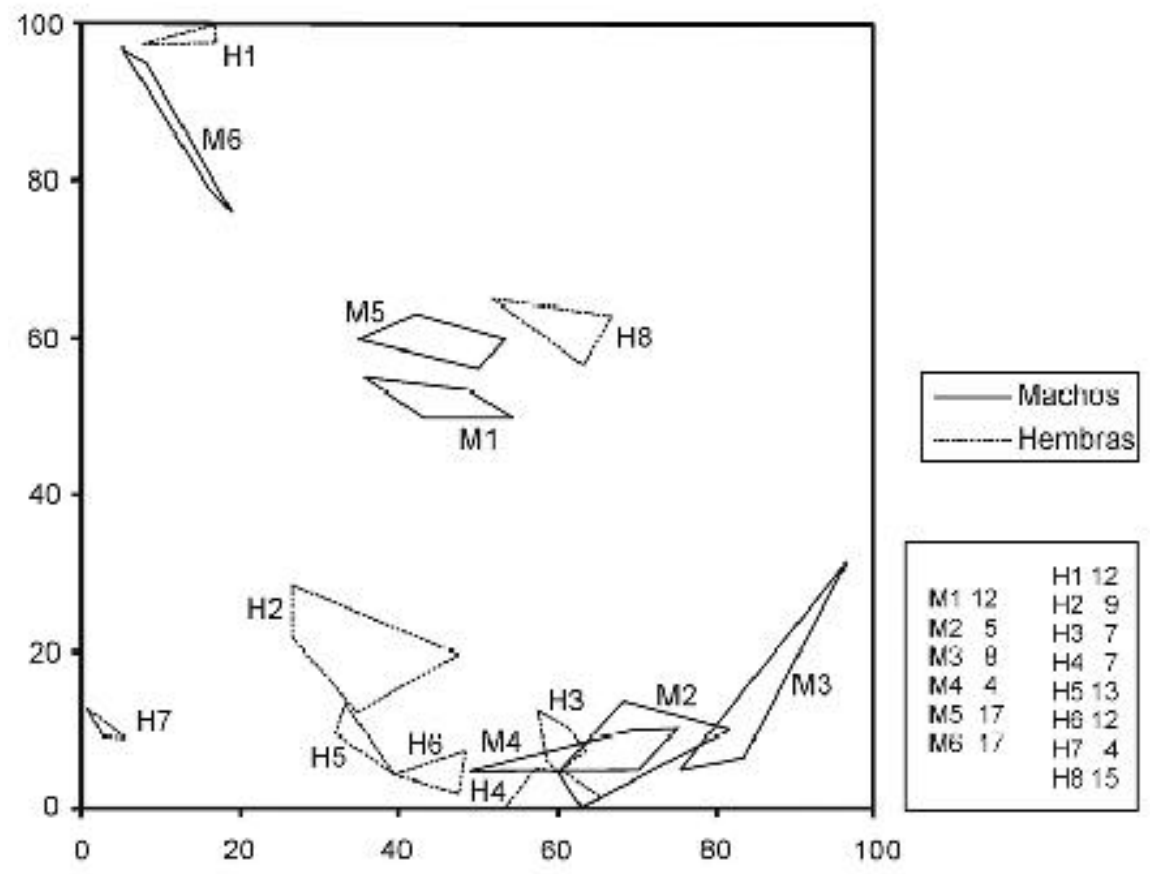

Figura 1: Ambito de hogar de machos (líneas completas: M) y hembras (líneas punteadas: H) de Liolaemus melanops. Se indica el número de observaciones en que se baso la estimación de cada ámbito de hogar.

FIGURE 1: Home range of males (full lines; $\mathrm{M}$ ) and females (dashed lines; $\mathrm{H}$ ) of Liolaemus melanops. The number of observations in which the home range estimations are based is indicated.

TABLA I: Solapamiento de ámbito de hogar entre pares de individuos de Liolaemus melanops. $\mathrm{H}=$ hembra; $\mathrm{M}=$ macho; los números de los individuos refieren a los usados en la Figura 1.

TABLE I: Home range overlaps between pairs of individuals of Liolaemus melanops. $\mathrm{H}=$ female; $\mathrm{M}=$ male; individual number refers to those used in Figure 1.

\begin{tabular}{lccc}
\hline Individuo A - B & $\begin{array}{c}\text { \% del ámbito de } \\
\text { hogar del individuo A } \\
\text { solapado al del individuo B }\end{array}$ & $\begin{array}{c}\text { \% del ámbito de } \\
\text { hogar del individuo B } \\
\text { solapado al del individuo A }\end{array}$ & $\begin{array}{c}\text { Area } \\
\text { Solapamiento } \\
\left(\mathrm{M}^{2}\right)\end{array}$ \\
\hline H3 - H4 & 0,15 & 0,085 & 0,04 \\
M2- M4 & 31,25 & 65,94 & 42,04 \\
M2- M3 & 1,87 & 2,76 & 2,52 \\
M4 - H3 & 11,73 & 20,08 & 7,48 \\
M2 - H4 & 4,67 & 13,39 & 6,28 \\
M2 - H3 & 0,29 & 1,5 & 0,4 \\
\hline
\end{tabular}




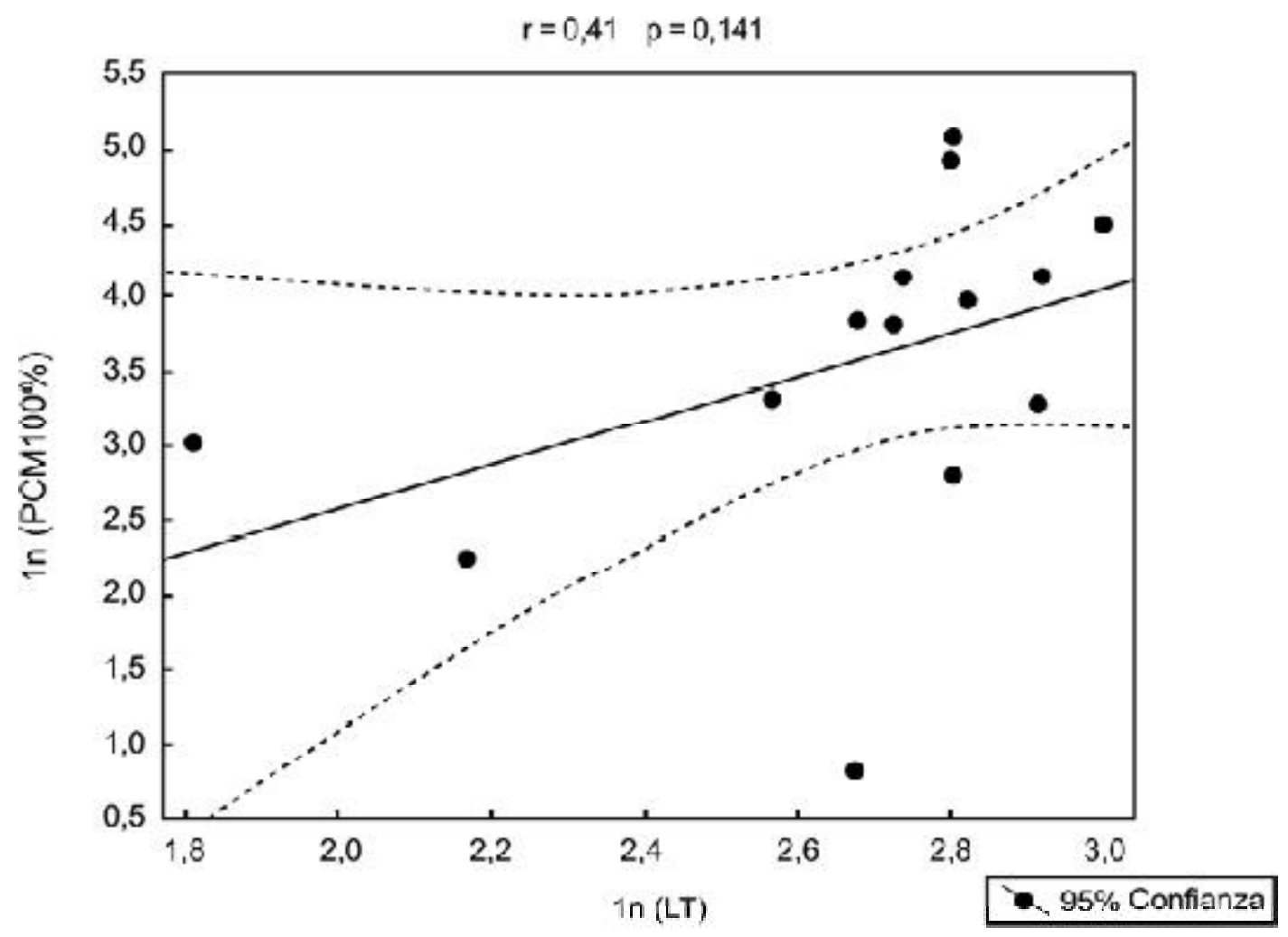

FIGURA 2: Correlación entre el tamaño del ámbito de hogar [ln (PCM100\%)] y el largo total [ln (LT)] de los individuos de Liolaemus melanops.

FIGURE 2: Correlation between home range size $[\ln (\mathrm{PCM} 100 \%)]$ and total length $[\ln (\mathrm{LT})]$ of Liolaemus melanops.

TABLA II: Longitud hocico-cloaca (LHC) y áreas de ámbito de hogar (Ah) de cuatro especies de Liolaemus.

TABLE II: Snout-cloaca length (LHC) and home range size (Ah) of four species of Liolaemus.

\begin{tabular}{|c|c|c|c|c|c|c|}
\hline & & Macho & Desv. Est. & Hembra & Desv. Est. & $\mathrm{AhM} / \mathrm{AhH}$ \\
\hline Liolaemus koslowskyi & LHC & $69,0 \mathrm{~mm}$ & $\pm 0,70$ & $64,0 \mathrm{~mm}$ & $\pm 1,08$ & \\
\hline Frutos 2001 & $\mathrm{Ah}$ & $140,0 \mathrm{~m}^{2}$ & $\pm 125,2$ & $25,0 \mathrm{~m}^{2}$ & $\pm 28,3$ & 5.6 \\
\hline Liolaemus lutzae & LHC & $78,0 \mathrm{~mm}$ & $\pm 3,0$ & $62,0 \mathrm{~mm}$ & $\pm 2,0$ & \\
\hline Rocha 1999 & $\mathrm{Ah}$ & $59,8 \mathrm{~m}^{2}$ & $\pm 33,7$ & $22,3 \mathrm{~m}^{2}$ & $\pm 16,1$ & 2.7 \\
\hline Liolaemus melanops & LHC & $76,4 \mathrm{~mm}$ & $\pm 0,64$ & $71,8 \mathrm{~mm}$ & $\pm 0,52$ & 1.6 \\
\hline Este estudio & $\mathrm{Ah}$ & $70,91 \mathrm{~m}^{2}$ & $\pm 37,4$ & $42,10 \mathrm{~m}^{2}$ & $\pm 51,3$ & (No sig.) \\
\hline Liolaemus quilmes & LHC & $61,3 \mathrm{~mm}$ & $\pm 0,48$ & $57,2 \mathrm{~mm}$ & $\pm 0,37$ & \\
\hline Halloy \& Robles 2002 & $\mathrm{Ah}$ & $132,2 \mathrm{~m}^{2}$ & $\pm 82,7$ & $29,2 \mathrm{~m}^{2}$ & $\pm 9,5$ & 4.5 \\
\hline
\end{tabular}




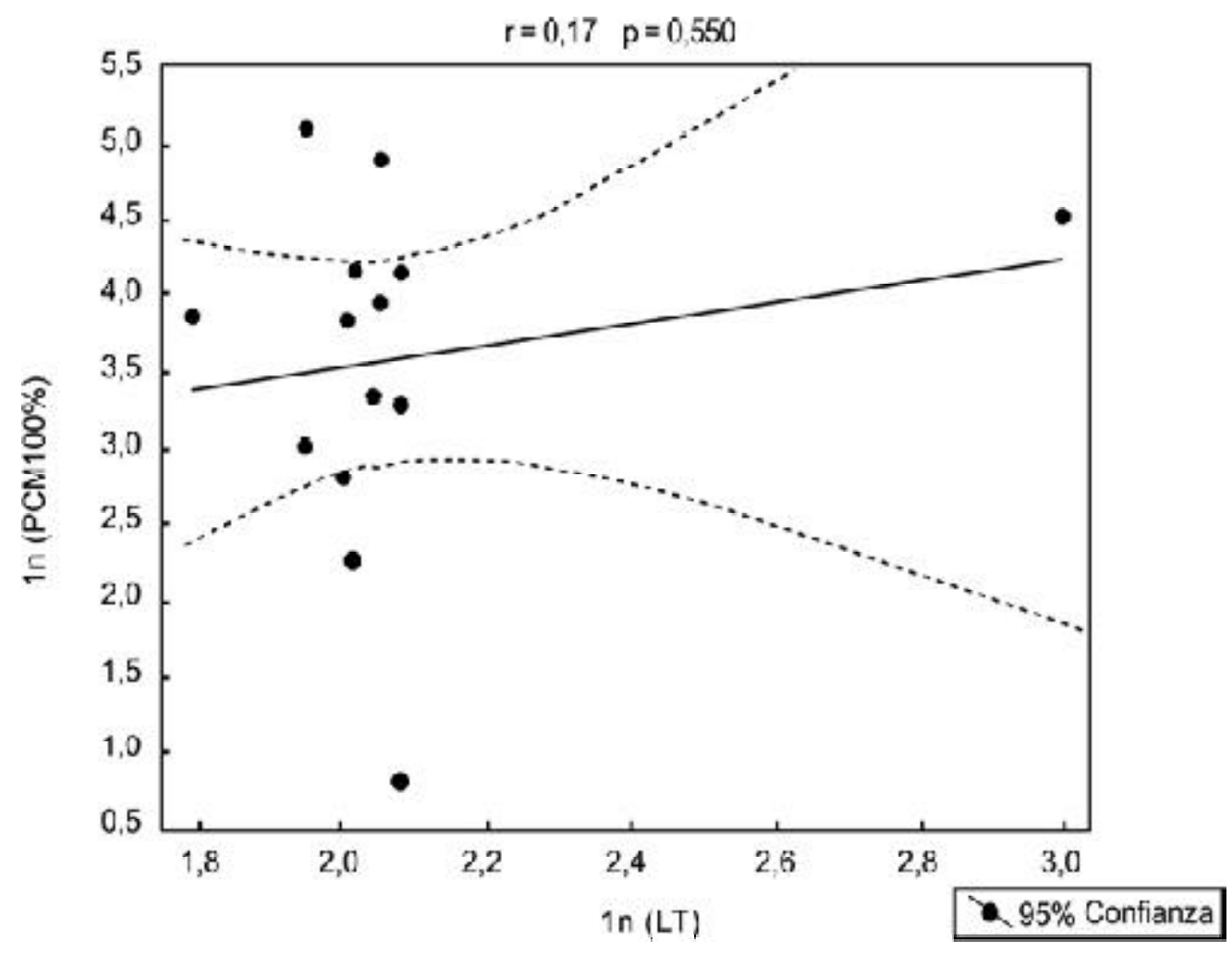

FiguRa 3: Correlación entre el tamaño del ámbito de hogar [ln (PCM100\%)] y el largo hocico-cloaca [ln (LHC)] de los individuos de Liolaemus melanops.

FIGURE 3: Correlation between home range size [ln (PCM100\%)] and snout-cloaca length [ln (LHC)] of Liolaemus melanops.

El hecho de no encontrar diferencias en los tamaños promedio del ámbito de hogar entre sexos de L. melanops, también podría deberse a que el estudio se realizo en la estación postreproductiva. Se ha demostrado que en algunas especies el tamaño del ámbito de hogar varía estacionalmente. Este es el caso de Anolis aeneus, Sauromalus obesus, Sceloporus jarrovis y Sceloporus virgatus cuyos ámbitos de hogar pasan de ser similares en ambos sexos en época no reproductiva a ser de 3,5 a 4 veces más grandes en los machos en época reproductiva (Stamps 1983). Por lo tanto, estudios adicionales en época reproductiva son necesarios para determinar si la falta de diferencias en los ámbitos de hogar de machos y hembra de L. melanopses constante a lo largo del año o es estacional.

El solapamiento observado entre los ámbitos de hogar de las hembras de L. melanops, al igual que en otras especies del género (L. quilmas: Halloy \& Robles 2002; L. koslowskyi: Frutos 2001), es mínimo. Por el contrario, en los machos el solapamiento fue importante. Este hecho reflejaría una baja defensa del territorio al menos durante esta época (período post reproductivo), sugiriendo la posibilidad de una utilización diferencial del territorio según la estación del año (Ferner 1974, Sheldhal \& Martins 2000, Wone \& Beauchamp 2003). En algunos iguánidos se ha reportado comportamientos territoriales en machos; sin embargo, se sugirió también la existencia de jerarquías en el caso en que los machos compartan dominios vitales (Halloy \& Robles 2002). En el caso de L. melanops son necesarios más estudios para clarificar a qué responde el gran solapamiento observado: falta de defensa estacional o un sistema de jerarquías que regule la convivencia. 
El solapamiento moderado entre machos y hembras podría ser un indicador del sistema de apareamiento, que en los lagartos va de la monogamia a distintos grado de poligamia (Stamps 1983). La posibilidad de que el ámbito de hogar de un macho se superponga al de varias hembras podría aumentar el éxito reproductivo de este; pero para ello, al mismo tiempo debe existir una defensa del territorio que permita monopolizar a las hembras del dominio (Perry \& Garland 2002, Wone \& Beauchamp 2003). En el caso de la especie focal se desconoce si el grado de solapamiento cambia en el período reproductivo; los machos podrían en esta época ampliar el ámbito de hogar para abarcar varias hembras, al tiempo que habría un cambio en el comportamiento territorial de los machos. EnL. koslowskyi la superposición entre el ámbito de hogar de machos y hembras fue mayor que dentro de cada uno de los sexos, en algunos casos el ámbito de hogar de dos o tres hembras estuvo incluido en el ámbito de hogar de un solo macho (Frutos 2001).

Si bien no se encontró correlación entre el ámbito de hogar y el tamaño corporal, biológicamente el tamaño de los individuos es un factor importante a la hora de explicar el tamaño del ámbito de hogar (Haenel et al 2003a, b), no obstante esta relación se ve más clara en análisis interespecíficos. A nivel intraespecífico el factor social puede ser más importante que las necesidades energéticas (Perry \& Garland 2001). Sin embargo, en Liolaemus melanops no se encontró esta relación positiva entre tamaño corporal y tamaño del ámbito de hogar. Esto concuerda con lo registrado para Liolaemuskoslowskyi (Frutos 2001). Ya que en L. melanops no se encontró diferencias en el tamaño del ámbito de hogar entre sexos en esta época, se deberían incluir en futuros estudios otros elementos como la heterogeneidad del ambiente, la densidad de presas, etc., para tener una idea más amplia acerca de la relación de las necesidades energéticas con el tamaño del ámbito de hogar.

\section{AGRADECIMIENTOS}

A los revisores anónimos, Dra. Mariana Morando, Dr. Néstor Basso, Dr. Héctor del Valle, Lic. Fernando Coronato y al Centro Nacional Patagónico (CENPAT -CONICET). Este trabajo fue apoyado por CONICET a través de un Proyecto de Investigación 6469/2004.

\section{BIBLIOGRAFIA}

Administración de Parques Nacionales/Secretaría de RecursosNaturalesy DesarrolloSustentable 1999. Ecorregiones de la Argentina. Buenos Aires. 42 p.

Araya Díaz, S. 2007. Aspectos autecológicos relevantes para la conservación de Phymaturus flagellifer (Reptilia, Tropiduridae) en la reserva nacional Altos de Lircay, Región del Maule. Memoria de Título. Universidad De Chile, Facultad deCiencias Agronómicas. Escuela de Agronomía. 40 pp.

Biotas $^{\mathrm{TM}}$. 2006. Ecological Software Solutions. Schwägalpstrasse 2, 9107 Urnäsch, Switzerland. Version 2.0a.

Brown, J.L. \& G.H. ORIANs. 1970. Spacing patterns in mobile animals. Annual Review in Ecology and Systematics 1:239-269.

BurT, W.H. 1943. Territoriality and Home Range concepts as applied to mammals. Journal of Mammalogy 24: 346-352.

Cei, J.M. 1986. Reptiles del centro, centro-oeste y sur de la Argentina. Herpetofauna de las zonas áridas y semiáridas. Museo Regionale di Scienze Naturali, Torino, Monografie IV: 527 pp.

Christian, K.A. \& S. WALDSChMidT. 1984. The relationship between lizards Home Range and body size: a reanalysis of the data. Herpetologica 40: 68-75.

FERNERJ. W. 1974. Home-range size and overlap in Sceloporus undulatus erythrocheilus (Reptilia: Iguanidae). Copeia 1974(2): 332-337.

Ferner, J.W. 1979. A Review Of Marking Techniques for Amphibians And Reptiles. Society For The Study Of Amphibians And Reptiles. Herpetological Circular 9. 42pp.

Fisher, M \& A. Muth. 1989. A technique for permanently marking lizards. Herpetological Review 20(2): 4546.

Fox S. F. \& P. A. ShipMAn. 2003. Social behavior at high and low elevations: environmental release and phylogenetic effects in Liolaemus: 310-355. En: Fox S. F.; J. K. McCoy, \& T. A. BAIRD (eds.). Lizard social behavior. John Hopkins University Press, New York. USA. 438 pp.

FruTos, N. 2001. Actividad diaria y Dominio Vital ("Home Range") de Liolaemus koslowskyi Etheridge, 1993 (Squamata: Iguania: Tropiduridae). Tesis de Licenciatura en ciencias biológicas. Facultad de Humanidades y Ciencias. Universidad Nacional del Litoral. Santa Fe, Argentina. 26 pp.

Fuentes, E. R. 1981. Depredadores, competidores y alimento como factores en la ecología de Liolaemus en Chile central. Medio Ambiente (Chile) 5: 22-27.

Fuentes E. R. \& CANCino, J. 1979. Rock-ground patchiness in a simple Liolaemus lizard community. Journal of Herpetology 13: 343-350.

GIL, M., V.Pérez Mellado \& F.G uerRero .1989.Estimación de dominios vitales en anfibios y reptiles. Metodología de muestreo y análisis de datos. Revista Española de Herpetología 3(2): 275-286. 
Habit, E. M. \& Ortiz J. C. 1994. Ambito de hogar de Phymaturus flagellifer (Reptilia: Tropiduridae). Boletín de la Sociedad de Biología de Concepción (Chile) 65: 149-152.

HaEnel, G.J.,L.C. SMith \& H.B. John-Alder. 2003a.HomeRange analysis in Sceloporus undulatus (Eastern Fence Lizard). I. Spacing patterns and the context of territorial behavior. Copeia: 2003. 99-102.

Haenel, G.J,L.C. Smith \& H.B. John-Alder. 2003b. HomeRange analysis in Sceloporus undulatus (Eastern Fence Lizard). II. A test of spatial relationships and reproductive success. Copeia 2003: 113-123.

Halloy, M. 1996. Behavioral patterns in Liolaemus quilmes (Tropiduridae), a Southe American Lizard. Bulletin of Maryland Herpetological Society 32: 43-57.

Halloy, M. \& C. Robles. 2002. Spatial distribution in a neotropical lizard, Liolaemusquilmes (Liolaemidae): site fidelity and overlapping among males and females. Bulletin of Maryland Herpetological Society 38: 118-129.

HAYNE, D.W. 1949. Calculation of size of home range. Journal of Mammalogy 30: 1-18.

Huey, R.B., E.R. Pianka \& T.W. Schoener. 1983. Lizard Ecology. Studies of a Model Organism. Harvard University Press, Cambridge, Massachusetts 501 pp.

Huey, R.B., C.R. Peterson, S.J.Arnold y W.P. Porter. 1989. Hot rocks and not-so-hot rocks: retreat-site selection by garter snakes and its thermal consequences. Ecology 70: 931-944.

INFOSTAT. 2005. InfoStat versión 2005. Grupo InfoStat, FCA, Universidad Nacional de Córdoba, Argentina.

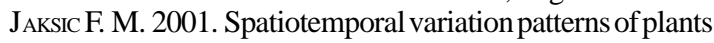
and animals in San Carlos de Apoquindo, central Chile. Revista Chilena de Historia Natural 74: 477502.

JAKSIC, F.M., H. NúÑEZ \& F. P. OJEDA. 1979. Proporciones corporales y utilización del hábitat en doce especies de Liolaemus de Chile central (Lacertilia, Iguanidae). Anales del Museo de Historia Natural de Valparaíso (Chile) 12: 59-68.

Jaksic, F. M., H. Núñez \& F. P. Ojeda. 1980. Body proportions, microhabitat selection, and adaptive radiation of Liolaemus lizards in central Chile. Oecologia 45: 178-181

Jerez, V. \& J. C. Ortiz. 1975. Distribución altitudinal del género Liolaemus (Squamata, Iguanidae) en el cerro La Campana (Parque Nacional La Campana). Anales del Museo de Historia Natural de Valparaíso (Chile) 8: 58-61.

Lovich, J.E. \& J.W. GibBons. 1992. A review of techniques for quantifying sexual dimorphism. Growth, Development Aging. 56: 269-281.

Manzur, M. I. \& E. R. Fuentes. 1979. Polygyny and agonistic behavior in the tree-dwelling lizard Liolaemus tenuis (Iguanidae). Behavioral Ecology and Sociobiology 6: 23-28.
Martins, E.P. 1994. Phylogenetic perspectives on the evolution of lizard territoriality. En: Lizard Ecology: historical and experimental perspectives. (Eds. Vitt, LJ \& ER Pianka). PUP, Princeton, New Jersey, USA.

M AURY, M.E. 1981. Variability of the activity cycles in some species of lizards in the Bolson de Mapimi (Mexico). En: Ecology of the Chihuahuan Desert. (Eds. R. Barbault y G. Halffter).

Medel, R. G., P. A. Marquet \& F. M. Jaksic. 1988. Microhabitat shifts of lizards under different contexts of sympatry: a case study with South American Liolaemus. Oecologia 76: 567-569

Ortiz, J.C. 1981. Révision taxonomique et biologie des Liolaemus du groupe nigromaculatus (Squamata, Iguanidae). Thèse de Doctorat d'Etats Sciencies Naturelles, Université Paris VII, 438pp.

Perry, G \& T. Garland Jr. 2002. Lizard Home Ranges revisited: Effects of sex, body size, diet, habitat, and phylogeny. Ecology 83: 1870-1885.

Rocha, C.F.D. 1999. Home Range of the Tropidurid Lizard Liolaemus lutzae: Sexual and body size differences. Revista Brasileña de Biología 59(1): 125-130.

Rose, B. 1982. Lizard home ranges: methodology and functions. Journal of Herpetology 16: 253-269.

Schulte II, J.A., J. B. Losos, F. B. CRuz \& H. Nuñez. 2004. The relationship between morphology, escape behaviour and microhabitat occupation in the lizard clade Liolaemus (Iguanidae: Tropidurinae: Liolaemini). Journal of Evolutionary Biology 17: 408-420.

ShedAhl, L. A. \& E. P. Martins. 2000. Territorial behavior in the western fence lizard. Herpetologica 56: 469479.

SimonetTi, J. 1984. Utilización de refugio por Liolaemus nigromaculatus: compromiso entre riesgos de predación y necesidades termorre-gulatorias. Studies on Neotropical Fauna and Environment 9: 47-51.

Simonetti, J. \& J. C. Ortiz. 1980. Dominio en Liolaemus kuhlmani (Reptilia: Iguanidae). Anales del Museo de Historia Natural de Valparaíso (Chile) 13: 167172.

STAMPS, J.A. 1983. Sexual selection, sexual dimorphism, and territoriality. En: Lizard Ecology. Studies of a Model Organism. (Eds. Huey, R.B., E.R. Pianka, y T.W. Schoener). HUP, Cambridge, MA, USA.

TelLeRIA, J.L. 1986. Manual para el censo de los vertebrados terrestres. Ed. Raices. Madrid. 278pp.

Turner, F.B., R.I. Jennrich \& J.D. Weintraub. 1969. Home ranges and body size lizards. Ecology 50: 10761081.

Watkins, G.C. 1996. Proximate causes of sexual size dimorphism in the iguanian lizard Microlophus occipitalis . Ecology. 77: 1473-1482.

Wone, B. \& B. Beauchamp. 2003. Movement, Home Range and activity patterns of the Horned Lizard, Phynosoma mcallii. Journal of Herpetology 37(4): 679-686. 